\title{
SPR-Measured Dissociation Kinetics of PROTAC Ternary Complexes Influence Target Degradation Rate
}

\author{
Michael J. Roy, ${ }^{\dagger}$ Sandra Winkler, ${ }^{\ddagger}$ Scott J. Hughes, ${ }^{\dagger}$ Claire Whitworth, ${ }^{\dagger}$ Michael Galant, ${ }^{\ddagger}$ \\ William Farnaby, ${ }^{\dagger}$ Klaus Rumpel, ${ }^{\dagger}$ and Alessio Ciulli*, ${ }^{\dagger}(0)$ \\ ${ }^{\dagger}$ Division of Biological Chemistry and Drug Discovery, School of Life Sciences, University of Dundee, James Black Centre, Dow \\ Street, Dundee DD1 5EH, United Kingdom \\ ${ }^{\ddagger}$ Boehringer Ingelheim RCV GmbH \& Co KG, 1221 Vienna, Austria
}

\section{Supporting Information}

ABSTRACT: Bifunctional degrader molecules, known as proteolysis-targeting chimeras (PROTACs), function by recruiting a target to an E3 ligase, forming a target/ PROTAC/ligase ternary complex. Despite the importance of this key intermediate species, no detailed validation of a method to directly determine binding parameters for ternary complex kinetics has been reported, and it remains to be addressed whether tuning the kinetics of PROTAC ternary complexes may be an effective strategy to improve the efficiency of targeted protein degradation. Here, we develop an SPR-based assay to quantify the stability of PROTACinduced ternary complexes by measuring for the first time the

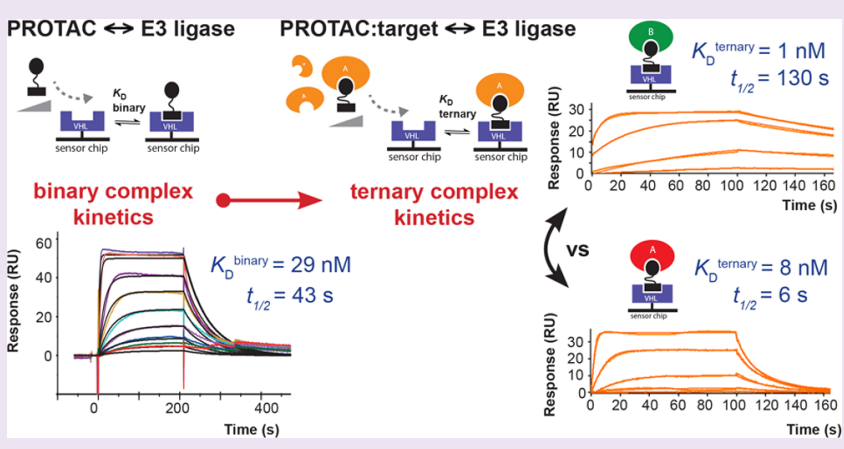
kinetics of their formation and dissociation in vitro using purified proteins. We benchmark our assay using four PROTACs that target the bromodomains (BDs) of bromodomain and extraterminal domain proteins Brd2, Brd3, and Brd4 to the von HippelLindau E3 ligase (VHL). We reveal marked differences in ternary complex off-rates for different PROTACs that exhibit either positive or negative cooperativity for ternary complex formation relative to binary binding. The positively cooperative degrader MZ1 forms comparatively stable and long-lived ternary complexes with either Brd4 ${ }^{\mathrm{BD} 2}$ or Brd2 ${ }^{\mathrm{BD} 2}$ and VHL. Equivalent complexes with $\mathrm{Brd} 3^{\mathrm{BD} 2}$ are destabilized due to a single amino acid difference (Glu/Gly swap) present in the bromodomain. We observe that this difference in ternary complex dissociative half-life correlates to a greater initial rate of intracellular degradation of $\mathrm{Brd} 2$ and Brd4 relative to Brd3. These findings establish a novel assay to measure the kinetics of PROTAC ternary complexes and elucidate the important kinetic parameters that drive effective target degradation.

$\mathrm{P}$ roteolysis-targeting chimeras (PROTACs) are bivalent molecules consisting of ligands for each of a target protein and an E3 ligase, joined via a linker. ${ }^{1,2}$ PROTAC behavior can be modeled by three-body binding equilibria. ${ }^{3}$ Formation of a target/PROTAC/ligase ternary complex triggers proximitydependent target protein ubiquitylation and degradation via the ubiquitin-proteasome system., ${ }^{2,4}$ PROTAC drug discovery is rapidly advancing in both academia and industry, fuelled by both improvement in drug-like properties and broader recognition of mechanistic advantages of degradation over inhibition. ${ }^{1,5,6}$ PROTACs offer potential for improved selectivity beyond that of the constituent target ligand by harnessing additional stabilizing or destabilizing de novo protein-protein or protein-linker interactions formed via the ternary complex. ${ }^{2,7-9}$ Thus, in the context of a PROTAC ternary complex $(A B C)$, the binding affinity of the PROTAC " $\mathrm{B}$ " to one protein partner " $\mathrm{C}$ " (binary binding) may be enhanced or reduced by the presence of the second protein partner "A" (ternary binding). This effect can be quantified in terms of a "cooperativity" $(\alpha)$ factor, defined as the ratio of binary and ternary dissociation constants for PROTAC binding to $\mathrm{C}\left(\alpha=K_{\mathrm{D}}{ }^{\text {binary }} / K_{\mathrm{D}}{ }^{\text {ternary }}\right)$ (Figure $\left.1 \mathrm{~A}\right) .^{2}$ Cooperativity may be described as "positive" ( $\alpha>1$, enhanced ternary binding affinity relative to binary, thus further stabilizing the complex), "negative" ( $\alpha<1$, reduced ternary binding affinity relative to binary, thus destabilizing the complex) or "noncooperative" ( $\alpha=1$, no change in binding affinity for $\mathrm{C}$ due to the presence of A). Developing new tools to understand cooperativity and avidity effects and ternary complex stability in PROTAC design is thus of significant interest. Although functional degraders can be generated in the absence of positive cooperativity, ${ }^{8,10}$ mounting evidence suggests enhancing cooperativity and stability of ternary complexes could be an effective strategy in PROTAC design. ${ }^{11-13}$ As bifunctional molecules, PROTACs are subject to a well-recognized "hook effect", whereby at high PROTAC concentrations, binary interactions may outcompete ternary complex formation. ${ }^{5,14} 3$ Cooperativity is expected to counter this "hook effect" often

Received: February 1, 2019

Accepted: February 5, 2019

Published: February 5, 2019 
A.

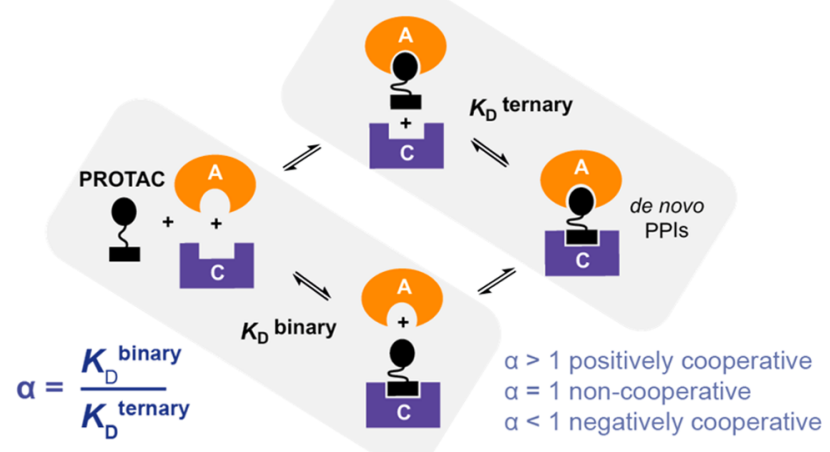

B. i) binding kinetics for PROTAC:VHL binary complex

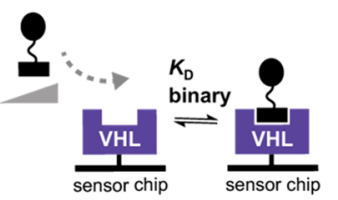

C. i) MZ1 binding to VHL (binary)
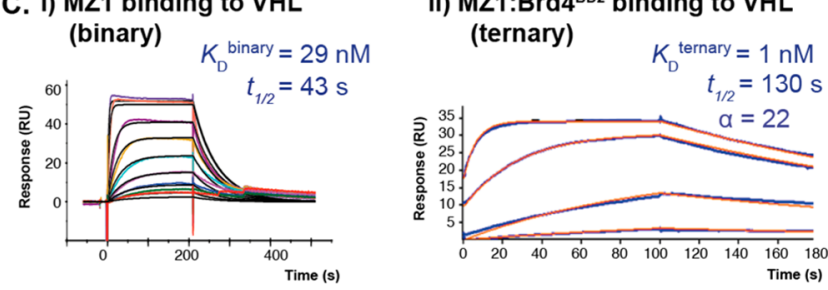

Figure 1. Schematic and binding data illustrating our SPR approach for measuring binding kinetics and determining cooperativity $(\alpha)$ for PROTAC binary and ternary complex formation. (A) Ternary binding equilibria, as occurs for bivalent molecules (such as PROTACs binding to two proteins, a target " $A$ " and an E3 ligase "C") may involve cooperativity effects, whereby the affinity of the bivalent molecule to one protein (binary complex formation) may be enhanced or decreased when it is already bound to the second protein (ternary complex formation). This may result from additional interactions present in the ternary complex, such as induced de novo protein-protein interactions (PPIs). This effect can be represented by a cooperativity factor $(\alpha)$, where $\alpha=K_{\mathrm{D}}{ }^{\text {binary }} / K_{\mathrm{D}}{ }^{\text {ternary }}$. (B) To measure the kinetics of PROTAC ternary complexes and determine cooperativity effects, we have developed an SPR assay in which we immobilized the E3 ligase (in our case, VHL) onto a sensor chip and measured binding of a PROTAC in either the (i) absence (binary binding) or (ii) presence (ternary binding) of near-saturating concentrations of the target protein (in our case, a bromodomain and extraterminal domain). (C) Representative SPR binding data are shown using this assay for (i) $\mathrm{MZ1}$ or (ii) the $\mathrm{MZ1} 1 \mathrm{Brd} 4^{\mathrm{BD} 2}$ complex binding to immobilized VHL. Binary and ternary binding experiments were performed at $285.15 \mathrm{~K}$ in multicycle kinetic format and $298.15 \mathrm{~K}$ in single-cycle kinetic (SCK) format, respectively. For each sensorgram, values shown represent fitted dissociation constants for binary or ternary complex formation $\left(K_{\mathrm{D}}{ }^{\text {binary }}\right.$ and $K_{\mathrm{D}}{ }^{\text {ternary }}$, respectively), dissociative half-life of the ternary complex $\left(t_{1 / 2}\right)$ (calculated as $t_{1 / 2}=$ $\ln 2 / k_{\text {off }}$ ), and cooperativity factor $(\alpha)$ (calculated as $\alpha=K_{\mathrm{D}}^{\text {binary }} /$ $\left.K_{\mathrm{D}}^{\text {ternary }}\right)$.

exhibited by bifunctional molecules, thereby widening the concentration window for PROTAC activity and could also enable the use of weaker-affinity ligands. ${ }^{15,16}$

There is now a growing literature of complementary methods to measure formation of PROTAC ternary complexes, offering different relative strengths and weaknesses (reviewed more comprehensively elsewhere). ${ }^{9}$ A number of steady-state methods have been described to measure ternary complex formation, cooperativity, or both. These include pulldown assays ${ }^{15}$ and proximity-based assays (e.g., AlphaScreen/ AlphaLISA, time-resolved fluorescence energy transfer (TRFRET)) to measure relative ternary complex formation at steady state as a function of PROTAC concentration. ${ }^{2,13,15,17}$ Such assays can offer high throughput but require the labeling of both target and E3 ligase proteins, and the readout of ternary complex formation is indirect and semi-quantitative, owing to context-specific effects that may potentially affect assay measurement and confound comparison between PROTACs (e.g., effects due to target protein identity, relative protein orientation in the ternary complex, concentrations of proteins used in the assay, and the intrinsic absorbance or fluorescence of PROTACs). Cooperativity assays based on competitive binding (e.g., fluorescence polarization, to measure PROTAC binding to VHL in the presence or absence of target protein) have also been used to determine binding and cooperativity of PROTACs. ${ }^{11}$ ITC has also been used for labelfree direct quantification of thermodynamic and binding parameters of PROTACs (for both binary and ternary complex formation), ${ }^{2,10,12}$ however, it is relatively more resourceintensive and lower throughput. Notably, none of these assays offer kinetic resolution. Recently, promising cell-based assays have been reported that enable real-time kinetic monitoring of PROTAC ternary complexes in cells, including the kinetic EGFP separation of phase-based protein interaction reporter $(\text { SPPIER })^{18}$ and kinetic bioluminescence resonance energy transfer (BRET) ${ }^{19}$ approaches. These cell-based methods enable the use of full-length proteins, albeit with the caveats that proteins must still be labeled (potentially giving rise to assay-specific effects described earlier), and the readout of ternary complex formation over time is still indirect. Promisingly, the ratiometric nature of BRET enables quantitative measurement. ${ }^{19}$ However, the readout in such cell-based assays may also be strongly affected by compoundspecific factors, including variable cell permeability or nonspecific binding to cellular components as well as the potential general complication of target protein degradation at later time points if the normal proteosomal pathway has not been disrupted either genetically or chemically. It is thus also desirable to have well-validated, quantitative biophysical assays to measure PROTAC ternary complex kinetics in vitro using purified proteins.

We were thus interested in exploring surface plasmon resonance (SPR) as a suitable label-free technique to monitor the kinetics of PROTAC-induced ternary complexes, the required intermediate species in the mechanism. ${ }^{4}$ Herein, we develop the first SPR-based assay to quantitatively measure the kinetics of ternary complex formation and dissociation, which we use to characterize the lifetime of ternary complexes composed of bromodomain-containing target proteins, PROTACs, and the von Hippel-Lindau E3 ligase (VHL).

SPR has previously been utilized to characterize three-body binding systems (including complexes composed of protein, DNA, and small molecules), ${ }^{20-23}$ which can be experimentally involving due to the complex nature of the binding equilibria. ${ }^{3}$ We sought a general-purpose and conceptually simple assay format to study PROTAC ternary complexes. Importantly, we recognized for bivalent molecules that the "hook effect" would preclude use of saturating concentrations of PROTAC in the running buffer. We reasoned that by immobilizing the E3 
ligase, a single sensor surface might be utilized to measure diverse PROTAC/target combinations. To improve uniform presentation on the chip surface, we designed a VHL/ ElonginB/ElonginC (VCB) construct harboring an AviTag sequence $\mathrm{C}$-terminal to ElonginB for site-specific biotinylation (hereafter "biotin-VHL"). ${ }^{24,25}$

Using a Biacore T200 SPR instrument and streptavidinimmobilized biotin-VHL, we measured the kinetics and affinity of VHL binding for a concentration series of either PROTAC alone (to form a binary complex with VHL, $K_{D}{ }^{\text {binary }}$ ) or PROTAC pre-incubated with near-saturating concentrations of target protein (to form a ternary complex with VHL, $K_{\mathrm{D}}{ }^{\text {ternary }}$; Figure 1$)$. To achieve near-saturation, the minimum concentration of target protein used was $2 \mu \mathrm{M}$ (corresponding to approximately a 20 - to 50 -fold excess of binary $K_{\mathrm{D}}$ of the PROTAC for the target protein to ensure at equilibrium $\geq 95-$ 98\% formation of binary complex) (refer to Figures S1 and S2). Experiments were performed in either multicycle (binary) or single-cycle (ternary) format without regeneration to ensure a maximally stable surface and reduce experimental run-times for PROTAC/BD complexes exhibiting slow dissociation kinetics (refer to Figure S3). ${ }^{26}$ Doubly referenced replicate data were fitted globally (over multiple surface densities where applicable) to a 1:1 Langmuir binding model incorporating a parameter for mass-transfer effects to determine kinetic constants $\left(k_{\text {on }}, k_{\text {off }}\right)$ from which dissociation constants were calculated $\left(K_{\mathrm{D}}=k_{\text {off }} / k_{\text {on }}\right)$. Cooperativity $(\alpha)$ was calculated as the ratio $K_{\mathrm{D}}^{\text {binary }} / K_{\mathrm{D}}$ ternary .

To benchmark the assay, we utilized the PROTAC MZ1, which forms a highly cooperative ternary complex with VHL and the second bromodomain $(\mathrm{BD} 2)$ of $\mathrm{Brd} 4\left(\mathrm{Brd} 4{ }^{\mathrm{BD} 2}\right)$ that we have previously characterized both biophysically and structurally. ${ }^{2,7,10}$ In addition to MZ1, we selected three other PROTACs with ITC-measured affinities for ternary complex formation with VHL/bromodomain and extraterminal domain (BET) bromodomains (see Chart 1). ${ }^{2,10}$ Combined, these

\section{Chart 1. PROTACs Utilized in This Study}
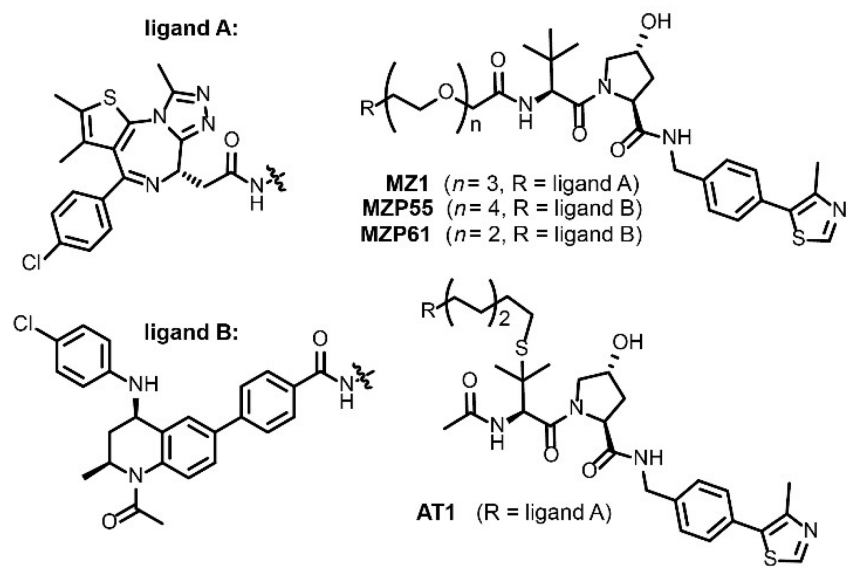

encompass a range of both binary binding affinities and ternary complex cooperativities (both positive and negative). This set includes MZ1 and AT1, two positively cooperative PROTACs based on a triazolodiazepine BET inhibitor JQ1 (ligand A, Chart 1), as well as MZP55 and MZP61, two negatively cooperative PROTACs based on a more potent tetrahydroisoquinolone BET inhibitor I-BET726 (ligand B, Chart 1).
SPR binding studies were performed with MZ1, AT1, MZP55, and MZP61 alone (binary) or in complex with $\mathrm{Brd} 4{ }^{\mathrm{BD} 2}$ as a representative BET bromodomain (ternary) and binding data compared with the ITC-obtained values (Tables 1 and S1 and Figures S1-S4). ${ }^{2,10}$ For MZ1, ternary complex formation was also measured with other BET bromodomains (Table 1 and Figure 2). No interaction was observed between VHL and $\mathrm{Brd} 4{ }^{\mathrm{BD} 2}$ in the absence of PROTAC (Figure S2). For the panel of PROTACs evaluated, the measured $K_{\mathrm{D}}$ values for binary and ternary complexes, as well as the calculated values for the both cooperativity and change in complex stability $(\Delta \Delta G)$, were remarkably comparable using either ITC or SPR (Table 1). For MZP61 and MZP55, some nonspecific effects were observed for binary binding to VHL (Figure S4); in the case of MZP61, these effects were sufficiently pronounced as to preclude accurate kinetic fitting, so steady-state fitting was used to estimate $K_{\mathrm{D}}^{\text {binary }}$.

Relative to the binary equilibria, the $\mathrm{VHL} / \mathrm{MZ1} / \mathrm{Brd} 4^{\mathrm{BD} 2}$ ternary complex displayed both a faster $k_{\text {on }}$ and a slower $k_{\text {off }}$ leading to the tighter $K_{\mathrm{D}}^{\text {ternary }}$ and significant positive cooperativity $(\alpha \approx 20)$ (see Figure $1 \mathrm{C}$ and Tables 1 and $\mathrm{S} 1)$. As anticipated, the VHL/AT $1 / \mathrm{Brd} 4^{\mathrm{BD} 2}$ ternary complex also exhibited positive cooperativity $(\alpha \approx 5)$. In stark contrast, ternary complexes formed by either of MZP55 and MZP61 with $\mathrm{Brd} 4{ }^{\mathrm{BD} 2}$ had very fast dissociation kinetics (>80-fold faster than the $\mathrm{VHL} / \mathrm{MZ} 1 / \mathrm{Brd} 4^{\mathrm{BD} 2}$ complex) reflecting overall negative cooperativity, with the ternary complex exhibiting the most negative cooperativity (relative to the binary binding to VHL) being that formed by MZP61 (Table S1 and Figure S4). Analogous experiments were conducted in a reversed format using immobilized $\mathrm{Brd} 4{ }^{\mathrm{BD} 2}$ and PROTAC in solution in the absence or presence of excess VHL. Although quantitative fitting of these ternary data was not possible, qualitatively, the sensorgrams reflected the same trends already described for the standard format (Figure S6). While we have shown that both the ITC and SPR approaches are complementary and yield similar values, the notable advantages of our SPR method are increased throughput and yielding kinetic information, including estimates of the lifetime of the ternary complex. This information is invaluable to better understand PROTAC function and ternary complex stability in an enzymatic context, $^{4}$ analogously to how quantification of inhibitor residence time has helped to understand pharmacological function in certain occupancy-driven small-molecule contexts. $^{27,28}$

MZ1 has been shown to degrade Brd4 more potently than Brd2 or Brd3 (despite near-equipotent binding of the constituent warhead ligand to all BET bromodomains), $7,10,19$ resulting from the high thermodynamic stability of the VCB/ $\mathrm{MZ1} / \mathrm{Brd}^{\mathrm{BD} 2}{ }^{\text {complex. }}{ }^{2}$ This data suggested varying levels of cooperativity for MZ1 VHL/PROTAC/BD ternary complexes. Moreover, literature data from us using biophysical methods ${ }^{2}$ and others using cellular degradation assays, ${ }^{8}$ as well as our own FP data (vide infra) are consistent with the MZ1 mediated degradation of BET proteins being driven by complex formation with the BD2s. In an effort to better understand on a kinetic level the basis for apparent differences in cooperativity, we sought to quantify the overall kinetics of VHL/PROTAC/BD ternary complexes for each of the different BET bromodomains (Figures 2, S7, and S8 and Tables 1 and S2). Ternary complexes consisting of VHL, MZ1, and the first bromodomain (BD1) of either $\operatorname{Brd} 2, \operatorname{Brd} 3$, or Brd4 all displayed very fast dissociation kinetics $\left(t_{1 / 2}<1 \mathrm{~s}\right)$, 
Table 1. Binding of PROTACs or PROTAC/BD Complexes to Immobilized VHL (SPR) and Comparison with ITC Data

\begin{tabular}{|c|c|c|c|c|c|c|c|c|c|c|c|}
\hline \multirow[b]{2}{*}{ PROTAC } & & \multirow[b]{2}{*}{+ target } & \multicolumn{6}{|c|}{$\operatorname{SPR}(\mathrm{VHL})^{a}$} & \multicolumn{3}{|c|}{ ITC $(\mathrm{VHL})^{2,10}$} \\
\hline & & & $K_{\mathrm{D}}(\mathrm{nM})$ & $k_{\mathrm{on}}\left(\mathrm{M}^{-1} \mathrm{~s}^{-1}\right) \times 10^{5}$ & $k_{\text {off }}\left(\mathrm{s}^{-1}\right)$ & $t_{1 / 2}(\mathrm{~s})$ & $\alpha$ & $\Delta \Delta G^{b}(\mathrm{kcal} / \mathrm{mol})$ & $K_{\mathrm{D}}(\mathrm{nM})$ & $\alpha$ & $\Delta \Delta G^{b}(\mathrm{kcal} / \mathrm{mol})$ \\
\hline \multirow[t]{7}{*}{ MZ1 } & binary & - & 29 & 7 & 0.019 & 43 & - & - & $66^{d}$ & - & - \\
\hline & ternary & $\operatorname{Brd} 2^{\mathrm{BD} 1}$ & 23 & 3900 & $>1$ & $<0.7$ & 1.3 & -0.1 & $24^{d}$ & $2.9^{d}$ & $-0.6^{d}$ \\
\hline & ternary & $\mathrm{Brd} 3^{\mathrm{BD} 1}$ & 12 & 1400 & $>1$ & $<0.7$ & 2.4 & -0.4 & $19^{d}$ & $3.5^{d}$ & $-0.8^{d}$ \\
\hline & ternary & $\mathrm{Brd} 4^{\mathrm{BD} 1}$ & 30 & 700 & $>1$ & $<0.7$ & 0.9 & +0.1 & $28^{d}$ & $2.3^{d}$ & $-0.5^{d}$ \\
\hline & ternary & $\mathrm{Brd} 2^{\mathrm{BD} 2}$ & 0.9 & 120 & 0.01 & 67.4 & 32 & -2.0 & $28^{d}$ & $2.3^{d}$ & $-0.5^{d}$ \\
\hline & ternary & $\mathrm{Brd} 3^{\mathrm{BD} 2}$ & 8 & 160 & 0.12 & 6 & 3.6 & -0.7 & $7^{d}$ & $10.7^{d}$ & $-1.5^{d}$ \\
\hline & ternary & $\mathrm{Brd} 4^{\mathrm{BD} 2}$ & 1 & 59 & 0.006 & 130 & 22 & -1.7 & $3.7^{d}$ & $17.8^{d}$ & $-1.7^{d}$ \\
\hline \multirow[t]{2}{*}{ AT1 } & binary & - & 110 & 6 & 0.06 & 17 & - & - & $335^{d}$ & - & - \\
\hline & ternary & $\mathrm{Brd} 4^{\mathrm{BD} 2}$ & 24 & 14 & 0.03 & - & 4.7 & -0.8 & $46^{d}$ & $7.3^{d}$ & $-1.2^{d}$ \\
\hline \multirow[t]{2}{*}{ MZP55 } & binary & - & 69 & 2.7 & 0.015 & 48 & - & - & $109^{e}$ & - & - \\
\hline & ternary & $\mathrm{Brd} 4^{\mathrm{BD} 2}$ & 185 & 27 & 0.47 & 1 & 0.4 & +0.7 & $183^{e}$ & $0.6^{e}$ & $+0.3^{e}$ \\
\hline \multirow[t]{2}{*}{ MZP61 } & binary & - & $104^{c}$ & - & - & - & - & - & $116^{e}$ & - & - \\
\hline & ternary & $\mathrm{Brd} 4^{\mathrm{BD} 2}$ & 465 & 30 & 1 & 1 & 0.2 & +1.0 & $781^{e}$ & $0.1^{e}$ & $+1.1^{e}$ \\
\hline
\end{tabular}

${ }^{a}$ Refer to Tables S1 and S2 for full details (including errors). All binary binding experiments using immobilized VHL were performed at $285.15 \mathrm{~K}$ in multicycle kinetic format, whereas ternary binding experiments were performed at $298.15 \mathrm{~K}$ in single-cycle kinetic (SCK) format (to decrease experimental run times for complexes that dissociated slowly). For ease of presentation and comparison, SCK data were overlaid in a format similar with multicycle kinetic data (as described in Figure S3). Representative sensorgrams for binary and ternary experiments are shown in Figures S4 and S7. For SPR data, listed values were calculated from fitted kinetic data as follows: dissociation constant $\left(K_{\mathrm{D}}=k_{\text {off }} / k_{\text {on }}\right)$, dissociative half-life $\left(t_{1 / 2}\right.$ $\left.=\ln 2 / k_{\text {off }}\right)$, and cooperativity $\left(\alpha=K_{\mathrm{D}}^{\text {binary }} / K_{\mathrm{D}}{ }^{\text {ternary }}\right)$; difference in standard Gibbs free energy change between ternary and binary binding $(\Delta \Delta G=$ $\left.\Delta G^{\text {ternary }}-\Delta G^{\text {binary }}\right)$ were given for which, in each case, $\Delta G=R T \ln K_{\mathrm{D}}$, where $K_{\mathrm{D}}$ is the appropriate binary or ternary dissociation constant (in $M$, although in reality, it is dimensionless), $R$ is the ideal gas constant $\left(R=1.9872 \mathrm{cal}^{-1} \mathrm{Kol}^{-1}\right)$, and $T$ is the experimental temperature (in $\left.\mathrm{K}\right)$. For MZP55 and MZP61 nonspecific effects were observed during injection; hence, these binary $K_{\mathrm{D}}$ values and kinetic constants may be considered approximate. ${ }^{b}$ Difference in standard Gibbs free energy change between ternary and binary binding $\left(\Delta \Delta G=\Delta G^{\text {ternary }}-\Delta G^{\text {binary }}\right)$; calculated for ITC data as described for SPR data. ${ }^{c}$ Fitting by steady-state affinity. ${ }^{d}$ Literature value (ref 2). ${ }^{e}$ Literature value (ref 10 ). Note: the off rates for $\mathrm{VHL} / \mathrm{MZ1} / \mathrm{BD} 1 \mathrm{~s}$ are too fast to be quantified using a Biacore T200 so are reported as above the upper limit of the instrument's typical working range $\left(k_{\text {off }}>1 \mathrm{~s}^{-1}\right)$.

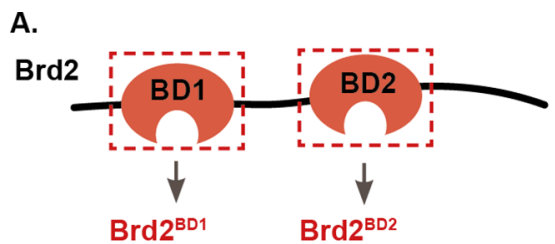

B. (i) $M Z 1: B r d 2^{B D 1}$



(ii) $\mathrm{MZ1}: \mathrm{Brd} 2^{\mathrm{BD} 2}$

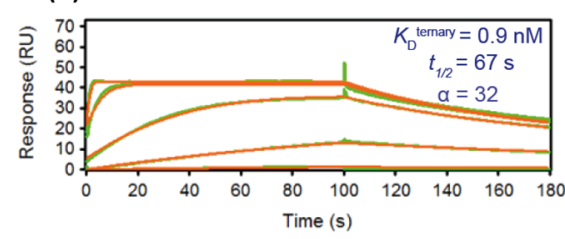

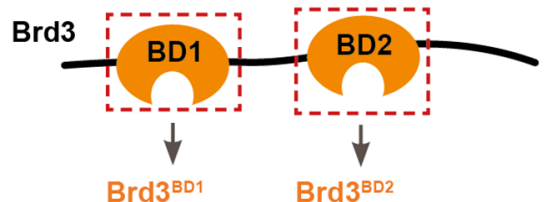

C. (i) $M Z 1: B r d 3^{B D 1}$

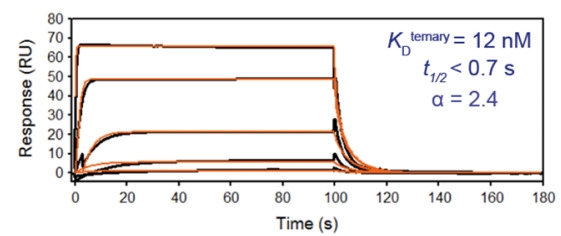

(ii) MZ1:Brd3 $3^{\mathrm{BD} 2}$

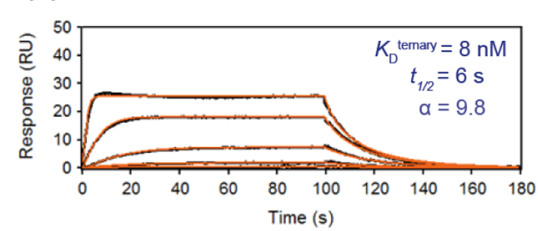

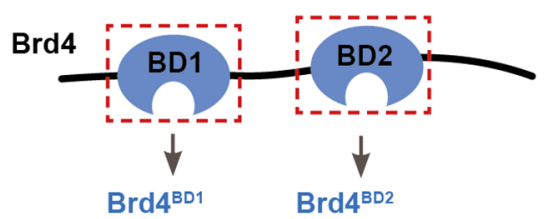

D. (i) $M Z 1: B r d 4$ BD1

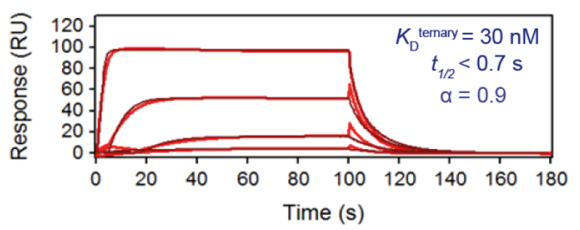

(ii) $\mathrm{MZ1}: \mathrm{Brd} 4^{\mathrm{BD} 2}$

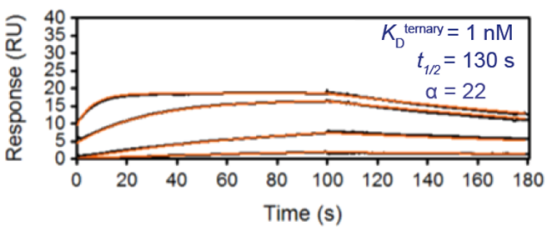

Figure 2. (A) Diagram of BET proteins Brd2, Brd3, and Brd4, showing individual bromodomains (BDs) used in this study; these BET proteins exhibit different degradation profiles in response to MZ1 treatment. ${ }^{2,7}$ To study the relative kinetics and cooperativity for ternary complex formation with MZ1 and VHL, MZ1/BD complexes were prepared and binding to immobilized VHL measured by SPR. (B) SPR sensorgrams for different MZ1/BD complexes reveal marked differences in binding kinetics, particularly VHL/MZ1/Brd2 ${ }^{\mathrm{BD} 2}$ and $\mathrm{VHL} / \mathrm{MZ1} / \mathrm{Brd} 4^{\mathrm{BD} 2}$ ternary complexes dissociated relatively slowly (as a result of the high positive cooperativity, $\alpha$, and greater complex stability). Ternary binding experiments were performed at $298.15 \mathrm{~K}$ in single-cycle kinetic (SCK) format. For each sensorgram, values shown represent fitted dissociation constants for ternary complex formation $\left(K_{\mathrm{D}}^{\text {ternary }}\right)$, dissociative half-life of the ternary complex $\left(t_{1 / 2}\right)$ (calculated as $\left.t_{1 / 2}=\ln 2 / k_{\text {off }}\right)$ and cooperativity factor $(\alpha)$ (calculated as $\left.\alpha=K_{\mathrm{D}}^{\text {binary }} / K_{\mathrm{D}}^{\text {ternary }}\right)$.

resulting overall in either low positive cooperativity or no cooperativity $(\alpha \approx 1)$. In the case of the second bromodomains (BD2), ternary complexes instead exhibited much-slower dissociation kinetics (up to 800 slower for a BD2 
compared to a $\mathrm{BD} 1$ in the case of $\mathrm{Brd} 2$; see Table 1 ), consistent with the known degradation-driving complexes (with BD2s) being the more-stable and longer-lived complexes. Crucially, we were struck by the significantly longer ternary half-life of the $\mathrm{VCB} / \mathrm{MZ1} / \mathrm{BD}$ ternary complexes with $\mathrm{Brd} 2^{\mathrm{BD} 2}$ and $\mathrm{Brd} 4^{\mathrm{BD} 2}\left(t_{1 / 2} \approx 70\right.$ and $130 \mathrm{~s}$, respectively $)$ relative to the more short-lived ternary complex with $\mathrm{Brd} 3^{\mathrm{BD} 2}$ $\left(t_{1 / 2} \approx 6 \mathrm{~s}\right)$.

Overlay of crystal structures of either Brd2 ${ }^{\mathrm{BD} 2}$ (PDB: 3ONI) or Brd3 ${ }^{\mathrm{BD} 2}$ (PDB: 3S92) in complex with JQ1 and the VCB/ $\mathrm{MZ1} / \mathrm{Brd} 4{ }^{\mathrm{BD} 2}$ structure (PDB: $\left.5 \mathrm{~T} 35\right){ }^{2}$ suggested that ternary complex formation could be influenced by a single amino acid residue difference ( $\mathrm{Glu}^{344}$ within the $\mathrm{ZA}$ loop of $\mathrm{Brd} 3^{\mathrm{BD} 2}$, which corresponds to $\mathrm{Gly}^{382}$ and $\mathrm{Gly}^{386}$ in $\mathrm{Brd} 2^{\mathrm{BD} 2}$ and $\mathrm{Brd} 4^{\mathrm{BD} 2}$ ) (Figure $3 \mathrm{~A}$ ). In an equivalent $\mathrm{VCB} / \mathrm{MZ} 1 / \mathrm{Brd} 3^{\mathrm{BD} 2}$ complex, the side-chain of $\mathrm{Glu}^{344}$ would induce severe steric clash with the VHL/MZ1 portion of the complex, leading to destabilization. We therefore generated reciprocal point mutant swaps (Figure 3B) and measured ternary complex formation by SPR with MZ1 or AT1. In all cases, the resulting SPR binding profiles reflected the effect predicted for the point mutation. The G-to-E point mutation in $\mathrm{Brd} 4^{\mathrm{BD} 2}$ or $\mathrm{Brd} 22^{\mathrm{BD} 2}$ shortened the ternary complex half-life, decreasing cooperativity and complex stability; while the reverse point mutation in $\mathrm{Brd} 3^{\mathrm{BD} 2}$ extended the ternary half-life to resemble the profile for $\mathrm{Brd} 4^{\mathrm{BD} 2}$ and, correspondingly, increased cooperativity and stability (Figures 3C and S9 and Table S2). As a crossvalidation, we evaluated these complexes in a competitive fluorescence polarization (FP) assay; measuring VHL binding of PROTAC or a PROTAC:BD binary complex via the displacement of a fluorescent HIF- $1 \alpha$ peptide probe (Table S2 and Figure S10). Good correlation was observed between SPRfitted dissociation constants $\left(K_{\mathrm{D}}\right)$ and FP-derived inhibition constants $\left(K_{\mathrm{I}}\right)$ (Figure $4 \mathrm{~A}$ ). Cooperativity values were also comparable using either method (Table S2). Together, these results underscore the robustness of our SPR approach and further support the conclusion that the described VCB/MZ1/ $\mathrm{Brd4}{ }^{\mathrm{BD} 2}$ structure (PDB: $\left.5 \mathrm{~T} 35\right)^{2}$ reflects the predominant (long-lived) species present in solution.

These SPR data illustrate kinetically a mechanistic difference between different PROTAC "archetypes". ${ }^{10}$ On the one hand, MZP55 and MZP61 are PROTACs with high binary target affinity (for Brd4) but low or negative cooperativity (Figures S4 and S6), thus likely forming highly populated binary complexes but a very transient ternary complex. On the other hand, MZ1 and AT1 exhibit weaker binary target affinity (for Brd4), but this is compensated for in the case of $\mathrm{Brd} 4{ }^{\mathrm{BD} 2}$ by significant positive cooperativity (to form stable ternary complexes). This latter case is predicted to fit a "rapid equilibrium" kinetics model, where a rate-limiting ubiquitination step is dependent on the concentration of PROTACinduced ternary complex. ${ }^{4}$ In this regime, an extension in ternary complex stability (and, hence, lifetime) would be expected to increase rates of target protein ubiquitination and degradation, particularly at early time points prior to countervailing factors such as protein resynthesis or feedback mechanisms. An alternative scenario that might be envisaged for PROTAC molecules operating under a different regime, e.g., in a "slow-binding regime", is that their association kinetics might be sufficiently rate limiting as to influence the ultimate outcome of the target degradation.

We wished to examine these hypotheses in a cellular context. Time-course studies were performed to measure initial rate of
A. i)
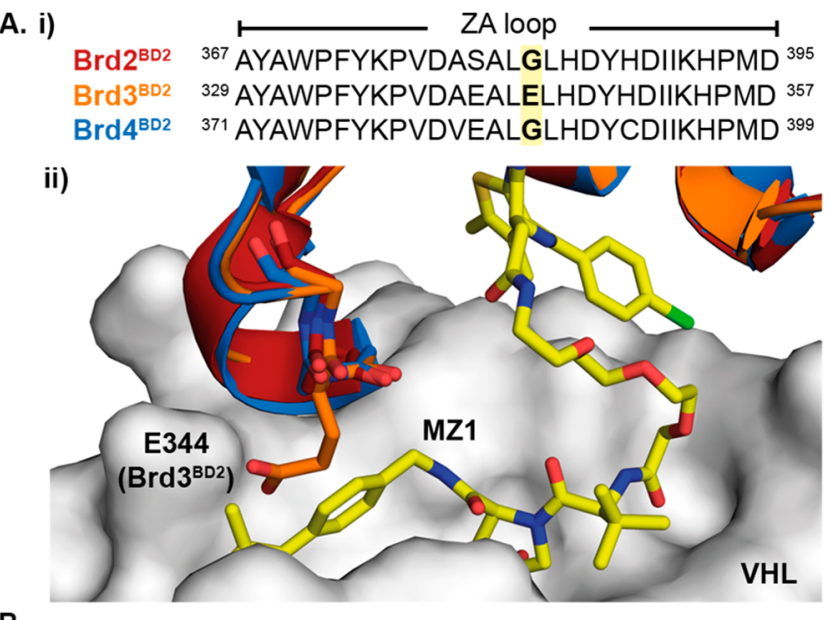

B.
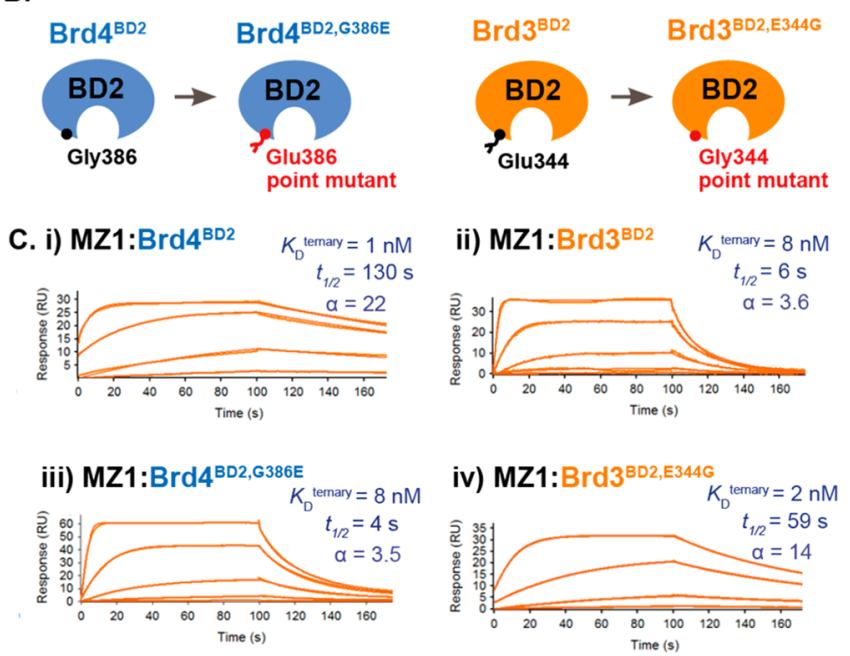

Figure 3. (A) Overlay of $\mathrm{Brd} 2^{\mathrm{BD} 2}$ (PDB: $3 \mathrm{ONI}$ ) and $\mathrm{Brd} 3^{\mathrm{BD} 2}$ (PDB: $3 \mathrm{~S} 92$ ) with the crystal structure of the $\mathrm{VCB} / \mathrm{MZ1} / \mathrm{Brd} 4^{\mathrm{BD} 2}$ ternary complex (PDB: 5T35) suggests that a $\mathrm{VHL} / \mathrm{MZ1} / \mathrm{Brd}^{\mathrm{BD} 2}$ ternary complex adopting the equivalent close-packing interaction would likely be less stable due to steric clash with the VHL/MZ1 of a single amino acid within the ZA loop of the bromodomain (Glu344 of $\mathrm{Brd} 3^{\mathrm{BD} 2}$, which corresponds to Gly386 in $\mathrm{Brd} 4^{\mathrm{BD} 2}$ ). (B) Diagram of point mutants generated to explore reciprocal swap of the amino acid at this position. (C) Reciprocal exchange of this single Gly/Glu residue in $\mathrm{Brd} 4^{\mathrm{BD} 2}$ (i, iii) or $\mathrm{Brd}^{\mathrm{BD} 2}$ (ii, iv) yields a corresponding swap of the kinetic profile in the resulting VHL/MZ1/BD ternary complex SPR sensorgram. For each sensorgram, values shown represent fitted dissociation constants for ternary complex formation $\left(K_{\mathrm{D}}{ }^{\text {ternary }}\right)$, dissociative half-life of the ternary complex $\left(t_{1 / 2}\right)$ (calculated as $t_{1 / 2}=\ln 2 / k_{\text {off }}$ ) and cooperativity factor $(\alpha)$ (calculated as $\left.\alpha=K_{\mathrm{D}}{ }^{\text {binary }} / K_{\mathrm{D}}{ }^{\text {ternary }}\right)$.

degradation of $\mathrm{Brd} 4, \mathrm{Brd} 3$, or $\mathrm{Brd} 2$ in response to treatment of HEK293 cells with MZ1 (Figure 4B; see Figure S11 for representative Western blot data). We observed rapid degradation of $\mathrm{Brd} 2$ and both isoforms of $\mathrm{Brd} 4$, while the degradation of Brd3 was significantly slower (Figure $4 \mathrm{~B}$ and Table S3). Recent results by an independent group, published while this manuscript was in preparation, strongly support our conclusion. ${ }^{19}$ Consistent with our data, Riching et al. observed more rapid initial rates of degradation and greater levels of ubiquitination for $\mathrm{Brd} 2$ and $\mathrm{Brd} 4$ compared to $\mathrm{Brd} 3$ in response to MZ1 treatment. ${ }^{19}$ Crucially, we observed a correlation between the half-life of the VHL/MZ1/BD2 ternary complexes and the initial rate of degradation of the 
A.


Figure 4. (A) Correlation between binary (MZ1) and ternary (MZ1/ $\mathrm{BD}$ complex) binding to VHL via SPR or FP (mean plus or minus the standard deviation, SD). (B) Initial degradation profile for BET proteins in HEK293 cells in response to MZ1 treatment (333 nM), with initial degradation rates $(\chi)$ estimated from data fitting (mean plus or minus the standard error of the mean, $N=3$ ) and SPR ternary half-lives for corresponding VHL/MZ1/BD2 complexes (mean $\pm \mathrm{SD}$ for $N=2$ ). Note that short and long isoforms of Brd4 differ in the length of the C-terminus after the tandem bromodomains. ${ }^{29}$

corresponding BET protein, with the more slowly degraded BET protein (Brd3, $\chi=4 \times 10^{3} \mathrm{~min}^{-1}, c f$. with $\mathrm{Brd} 2$ and $\mathrm{Brd} 4$, which show $\chi$ between 13 and $18 \times 10^{3} \mathrm{~min}^{-1}$ ) being also the one that exhibits the shortest-lived ternary complex (Figure 4B). This data is consistent with the rapid equilibrium framework, with the more stable and more slowly dissociating ternary complexes driving cellular protein degradation, and not consistent with a slow-binding ternary complex model; ${ }^{4}$ indeed, $\mathrm{MZ} 1 / \mathrm{Brd} 3^{\mathrm{BD} 2}$ showed comparatively fast association kinetics in our SPR data for binding to VHL $\left(k_{\text {on }}=160 \times 10^{5}\right.$ $\mathrm{M}^{-1} \mathrm{~s}^{-1}$ ), possibly even slightly faster as compared to MZ1/ $\mathrm{Brd} 2^{\mathrm{BD} 2}$ and $\mathrm{MZ} 1 / \mathrm{Brd} 4^{\mathrm{BD} 2}$ (Table 1 ). Taken together, these observations strongly suggest a mechanistic link between the relative half-life of a given target/PROTAC/E3 ligase ternary complex and initial rates of target degradation, which drives a faster and more profound target depletion in cells, at least for PROTACs operating under the "rapid equilibrium" kinetics model such as the archetypical degrader MZ1 studied herein.

In conclusion, we demonstrate a simple and robust SPRbased method to quantify for the first time the stability of
target/PROTAC/ligase ternary complexes by measuring the kinetics of their formation and dissociation. We demonstrate that our surface-based SPR method yields values for affinity, cooperativity $(\alpha)$ and complex stability comparable to ITC in solution ${ }^{2,10}$ with increased throughput and yielding additional kinetic information not achievable using other assays described to date. We show by SPR that a single residue can impart significant changes in cooperativity, stability, and dissociative half-lives of ternary complexes formed with different but highly conserved target proteins. Lastly, we observe that these kinetic differences of ternary complexes correlate to relative initial rates of target degradation for the well-characterized BET degrader MZ1. Together, these findings establish a new assay for PROTAC ternary complex kinetics and illuminate on ternary complex stability and dissociative half-lives as key optimization parameters for PROTAC design and discovery campaigns.

Our studies suggest that a potential challenge or limitation of this approach is the requirement for moderate quantities of whichever target protein is used in "near-saturating" concentrations; however, our experiments also suggest that meaningful data may still be obtained using lower concentrations than that generally used in this study (Figures S1 and S5). Our data also suggest that it may be necessary to evaluate both binding orientations (immobilizing either the target protein or E3 ligase on the sensor surface) to ascertain which format yields the best data. Nonspecific effects exhibited by certain PROTACs may also pose challenges in determining binary dissociation constants. This notwithstanding, we anticipate that our SPR kinetic assay will become an established tool to drive PROTAC development and to further elucidate the dynamic processes governing their mode of action. We envision that one natural extension of our approach may be adaptation to use in more high-throughput assays, in particular due to the additional kinetic information it provides relative to alternative binding approaches such as AlphaScreen or TRFRET. This is rendered more feasible by the availability of newer highly parallel SPR instruments with improved sensitivity, reducing sample consumption. In our described approach, the E3 ligase (in this case, VHL) is immobilized on the sensor surface, which has the advantage of being agnostic of the target protein of interest; using this format, any target protein (domain or full-length) might conceivably be used that is capable of recombinant production. In suitable cases, a reversed format using immobilized target protein might provide certain benefits, such as enabling the same PROTAC/E3 ligase solution to be simultaneously screened against both targets and anti-targets on parallel flowcells. Another possibility might be direct capture of over-expressed full-length protein from cell lysate onto the sensor surface by way of a suitable affinity tag. Furthermore, immobilization of the protein of interest may make this approach capable of extension to screening for the most-suitable E3 ligase/ PROTAC complexes for forming stable and long-lived complexes. Beyond PROTACs, this assay could also be applied more broadly to study three-body binding equilibria induced by other classes of heterobivalent molecules. ${ }^{30}$

\section{ASSOCIATED CONTENT}

\section{Supporting Information}

The Supporting Information is available free of charge on the ACS Publications website at DOI: 10.1021/acschembio.9b00092. 
Tables showing fitted SPR data, SPR and FP binding studies, and fitted degradation time-course data; figures showing the selection of the PROTAC-to-target ratio, lack of significant interaction, data treatment, SPR sensorgrams, the effect of varying the PROTAC-totarget ratio, reversed-format SPR binding experiments, fitted fluorescence polarization competition data, and a representative Western blot; additional details on experimental methods (PDF)

\section{AUTHOR INFORMATION}

\section{Corresponding Author}

*E-mail: a.ciulli@dundee.ac.uk.

\section{ORCID}

Alessio Ciulli: 0000-0002-8654-1670

\section{Funding}

This project has received funding from the European Research Council (ERC) under the European Union's Seventh Framework Programme (FP7/2007-2013) as a Starting Grant to A.C. (grant agreement no. ERC-2012-StG-311460 DrugE3CRLs), and by Boehringer Ingelheim. Biophysics and drug discovery activities at Dundee were supported by Wellcome Trust strategic awards (100476/Z/12/Z and 094090/Z/10/Z, respectively) to the Division of Biological Chemistry and Drug Discovery.

\section{Notes}

A preprint of this article (doi: 10.1101/451948) has been posted on bioRxiv: http://biorxiv.org/cgi/content/short/ $451948 \mathrm{v} 1$.

The authors declare the following competing financial interest(s): The Ciulli laboratory receives sponsored research support from Boehringer Ingelheim and Nurix, Inc. A.C. is a scientific founder, director, and shareholder of Amphista Therapeutics, a company that is developing targeted protein degradation therapeutic platforms.

\section{ACKNOWLEDGMENTS}

We thank chemists of the Ciulli group and at Boehringer Ingelheim for the gifts of compounds, K. H. Chan for discussions, G. Glendinning and S. Mayer for compound logistics, the Dundee MRC PPU Reagents and Services facility for the gift of the GST-BirA construct and for DNA sequencing services, and the Dundee Fingerprints Proteomics Facility for the ESI-MS analysis of protein samples.

\section{REFERENCES}

(1) Bondeson, D. P., and Crews, C. M. (2017) Targeted Protein Degradation by Small Molecules. Annu. Rev. Pharmacol. Toxicol. 57, 107-123.

(2) Gadd, M. S., Testa, A., Lucas, X., Chan, K. H., Chen, W., Lamont, D. J., Zengerle, M., and Ciulli, A. (2017) Structural basis of PROTAC cooperative recognition for selective protein degradation. Nat. Chem. Biol. 13, 514-521.

(3) Douglass, E. F., Jr., Miller, C. J., Sparer, G., Shapiro, H., and Spiegel, D. A. (2013) A comprehensive mathematical model for threebody binding equilibria. J. Am. Chem. Soc. 135, 6092-6099.

(4) Fisher, S. L., and Phillips, A. J. (2018) Targeted protein degradation and the enzymology of degraders. Curr. Opin. Chem. Biol. $44,47-55$.

(5) Churcher, I. (2018) Protac-Induced Protein Degradation in Drug Discovery: Breaking the Rules or Just Making New Ones? J. Med. Chem. 61, 444-452.
(6) Burslem, G. M., Smith, B. E., Lai, A. C., Jaime-Figueroa, S., McQuaid, D. C., Bondeson, D. P., Toure, M., Dong, H., Qian, Y., Wang, J., Crew, A. P., Hines, J., and Crews, C. M. (2018) The Advantages of Targeted Protein Degradation Over Inhibition: An RTK Case Study. Cell Chem. Biol. 25, 67-77.

(7) Zengerle, M., Chan, K. H., and Ciulli, A. (2015) Selective Small Molecule Induced Degradation of the BET Bromodomain Protein BRD4. ACS Chem. Biol. 10, 1770-1777.

(8) Nowak, R. P., DeAngelo, S. L., Buckley, D., He, Z., Donovan, K. A., An, J., Safaee, N., Jedrychowski, M. P., Ponthier, C. M., Ishoey, M., Zhang, T., Mancias, J. D., Gray, N. S., Bradner, J. E., and Fischer, E. S. (2018) Plasticity in binding confers selectivity in ligand-induced protein degradation. Nat. Chem. Biol. 14, 706.

(9) Hughes, S. J., and Ciulli, A. (2017) Molecular recognition of ternary complexes: a new dimension in the structure-guided design of chemical degraders. Essays Biochem. 61, 505-516.

(10) Chan, K. H., Zengerle, M., Testa, A., and Ciulli, A. (2018) Impact of Target Warhead and Linkage Vector on Inducing Protein Degradation: Comparison of Bromodomain and Extra-Terminal (BET) Degraders Derived from Triazolodiazepine (JQ1) and Tetrahydroquinoline (I-BET726) BET Inhibitor Scaffolds. J. Med. Chem. 61, 504-513.

(11) Zoppi, V., Hughes, S. J., Maniaci, C., Testa, A., Gmaschitz, T., Wieshofer, C., Koegl, M., Riching, K., Daniels, D. L., Spallarossa, A., and Ciulli, A. (2019) Iterative design and optimization of initially inactive Proteolysis Targeting Chimeras (PROTACs) identify VZ185 as a potent, fast and selective von Hippel-Lindau (VHL)-based dual degrader probe of BRD9 and BRD7. J. Med. Chem. 62, 699.

(12) Maniaci, C., Hughes, S. J., Testa, A., Chen, W., Lamont, D. J., Rocha, S., Alessi, D. R., Romeo, R., and Ciulli, A. (2017) HomoPROTACs: bivalent small-molecule dimerizers of the VHL E3 ubiquitin ligase to induce self-degradation. Nat. Commun. 8, 830.

(13) Zorba, A., Nguyen, C., Xu, Y., Starr, J., Borzilleri, K., Smith, J., Zhu, H., Farley, K. A., Ding, W., Schiemer, J., Feng, X., Chang, J. S., Uccello, D. P., Young, J. A., Garcia-Irrizary, C. N., Czabaniuk, L., Schuff, B., Oliver, R., Montgomery, J., Hayward, M. M., Coe, J., Chen, J., Niosi, M., Luthra, S., Shah, J. C., El-Kattan, A., Qiu, X., West, G. M., Noe, M. C., Shanmugasundaram, V., Gilbert, A. M., Brown, M. F., and Calabrese, M. F. (2018) Delineating the role of cooperativity in the design of potent PROTACs for BTK. Proc. Natl. Acad. Sci. U. S. A. 115, E7285-E7292.

(14) Raina, K., and Crews, C. M. (2017) Targeted protein knockdown using small molecule degraders. Curr. Opin. Chem. Biol. $39,46-53$.

(15) Bondeson, D. P., Smith, B. E., Burslem, G. M., Buhimschi, A. D., Hines, J., Jaime-Figueroa, S., Wang, J., Hamman, B. D., Ishchenko, A., and Crews, C. M. (2018) Lessons in PROTAC Design from Selective Degradation with a Promiscuous Warhead. Cell Chem. Biol. $25,78-87$.

(16) Testa, A., Lucas, X., Castro, G. V., Chan, K. H., Wright, J. E., Runcie, A. C., Gadd, M. S., Harrison, W. T. A., Ko, E. J., Fletcher, D., and Ciulli, A. (2018) 3-Fluoro-4-hydroxyprolines: Synthesis, Conformational Analysis, and Stereoselective Recognition by the VHL E3 Ubiquitin Ligase for Targeted Protein Degradation. J. Am. Chem. Soc. 140, 9299-9313.

(17) Winter, G. E., Mayer, A., Buckley, D. L., Erb, M. A., Roderick, J. E., Vittori, S., Reyes, J. M., di Iulio, J., Souza, A., Ott, C. J., Roberts, J. M., Zeid, R., Scott, T. G., Paulk, J., Lachance, K., Olson, C. M., Dastjerdi, S., Bauer, S., Lin, C. Y., Gray, N. S., Kelliher, M. A., Churchman, L. S., and Bradner, J. E. (2017) BET Bromodomain Proteins Function as Master Transcription Elongation Factors Independent of CDK9 Recruitment. Mol. Cell 67, 5-18.

(18) Chung, C. I., Zhang, Q., and Shu, X. (2018) Dynamic Imaging of Small Molecule Induced Protein-Protein Interactions in Living Cells with a Fluorophore Phase Transition Based Approach. Anal. Chem. 90, 14287-14293.

(19) Riching, K. M., Mahan, S., Corona, C. R., McDougall, M., Vasta, J. D., Robers, M. B., Urh, M., and Daniels, D. L. (2018) 
Quantitative Live-Cell Kinetic Degradation and Mechanistic Profiling of PROTAC Mode of Action. ACS Chem. Biol. 13, 2758-2770.

(20) Banaszynski, L. A., Liu, C. W., and Wandless, T. J. (2005) Characterization of the FKBP.rapamycin.FRB ternary complex. J. Am. Chem. Soc. 127, 4715-4721.

(21) Andersen, P. S., Schuck, P., Sundberg, E. J., Geisler, C., Karjalainen, K., and Mariuzza, R. A. (2002) Quantifying the energetics of cooperativity in a ternary protein complex. Biochemistry 41, 51775184.

(22) Palau, W., and Di Primo, C. (2012) Single-cycle kinetic analysis of ternary DNA complexes by surface plasmon resonance on a decaying surface. Biochimie 94, 1891-1899.

(23) Domenici, F., Frasconi, M., Mazzei, F., D’Orazi, G., Bizzarri, A. R., and Cannistraro, S. (2011) Azurin modulates the association of $\mathrm{Mdm} 2$ with p53: SPR evidence from interaction of the full-length proteins. J. Mol. Recognit. 24, 707-714.

(24) Fairhead, M., and Howarth, M. (2015) Site-specific biotinylation of purified proteins using BirA. Methods Mol. Biol. 1266, 171-184.

(25) Beckett, D., Kovaleva, E., and Schatz, P. J. (1999) A minimal peptide substrate in biotin holoenzyme synthetase-catalyzed biotinylation. Protein Sci. 8, 921-929.

(26) Karlsson, R., Katsamba, P. S., Nordin, H., Pol, E., and Myszka, D. G. (2006) Analyzing a kinetic titration series using affinity biosensors. Anal. Biochem. 349, 136-147.

(27) Copeland, R. A., Pompliano, D. L., and Meek, T. D. (2006) Drug-target residence time and its implications for lead optimization. Nat. Rev. Drug Discovery 5, 730-739.

(28) Copeland, R. A. (2016) The drug-target residence time model: a 10-year retrospective. Nat. Rev. Drug Discovery 15, 87-95.

(29) Conrad, R. J., Fozouni, P., Thomas, S., Sy, H., Zhang, Q., Zhou, M. M., and Ott, M. (2017) The Short Isoform of BRD4 Promotes HIV-1 Latency by Engaging Repressive SWI/SNF ChromatinRemodeling Complexes. Mol. Cell 67, 1001-1012.

(30) Costales, M. G., Matsumoto, Y., Velagapudi, S. P., and Disney, M. D. (2018) Small Molecule Targeted Recruitment of a Nuclease to RNA. J. Am. Chem. Soc. 140, 6741-6744. 